\title{
Maximizing the profit per unit of time for the TSP with convex resource-dependent travelling times
}

\author{
Moshe Zofi ${ }^{1,2 *}$, Ron Teller ${ }^{1}$ and Moshe Kaspi ${ }^{1}$ \\ ${ }^{1}$ Department of Industrial Engineering and Management, Ben-Gurion University of the Negev, Beer-Sheva, \\ Israel; and ${ }^{2}$ Department of Industrial Management, Sapir Academic College, Sderot, Israel
}

This paper introduces a new problem that is an extension of the travelling salesman problem (TSP) in which the travelling times are resource dependent and the objective is to maximize the profit per unit of time. We present an optimal solution approach comprised of three main steps: (1) calculating the optimal amount of total resource required (regardless of the selected tour); (2) constructing the tour; and (3) assigning the optimal resource to each connection between vertices using the equivalent load method. This solution approach finds the optimal solution with the same computational complexity for solving the classic TSP.

Journal of the Operational Research Society (2017) 68(10), 1177-1182. doi:10.1057/s41274-016-0156-5; published online 14 December 2016

Keywords: travelling salesman; resource allocation; optimization; profit per unit of time; equivalent load method

\section{Introduction}

The classic travelling salesman problem (TSP) is defined as follows: a salesman who is located in his home city is required to visit exactly once each city on a given list and to return home. The salesman must determine the order in which he visits the cities so that the total cost (time or distance) covered is minimized. The problem is modelled using a graph containing $n$ vertices representing the cities and $m$ weighted edges representing the travelling cost (time or distance) between the vertices they connect. The objective is to find a tour passing through all $n$ vertices that minimizes the total cost.

The TSP is a well-known NP-hard problem. It can be solved through optimal procedures, such as full enumeration, or by using more sophisticated procedures, such as cutting planes, branch and bound algorithms, or dynamic programming algorithms. To the best of our knowledge, the largest instance of the problem that has been solved optimally contains 85,900 vertices (Applegate et al, 2009). Alternatively, the TSP can be solved through approximation methods, which are more frequently used in practice. Solution procedures for the problem can be found in Hartley (1985), Lawler et al (1985), Papadimitriou and Steiglitz (1998), Williams (1999), Applegate et al (2007), Cook et al (2008), and Cook (2011).

There are many extensions to the classic TSP such as: (1) the TSP with profits (Feillet et al, 2005) where each vertex is associated with a profit and the salesman is not required to

*Correspondence: Moshe Zofi, Department of Industrial Engineering and Management, Ben-Gurion University of the Negev, Beer-Sheva, Israel. E-mail: zofi@bgu.ac.il visit all the vertices. His objective is to find the tour that yields the greatest profit. (2) The TSP with time windows (López-Ibáñez et al, 2013) in which the salesman must visit each of the vertices within a given time window, and the objective is to minimize the travelling time or the makespan. (3) The time-dependent TSP (Abeledo et al, 2013), in which the travelling cost of an edge depends on its position in the tour. (4) The maximum profit per unit time TSP (Kaspi et al, 2013) in which completing the tour provides a given predefined income $V$ and the objective is to find the tour that will maximize the ratio between the profit and the total travelling time.

One of the basic assumptions of the TSP is that the distance (or travel time or travel cost) between any pair of cities is known. However, in various real-life systems, processing (in manufacturing applications) or travelling times are controllable by using limited disposable resources such as financial budgets, overtime, energy, fuel, subcontracting or manpower. In most cases, increasing the amount of resource will reduce the travelling time at a decreasing rate.

This paper introduces a new extension of the TSP in which the travelling times are resource dependent and the total amount of resource is limited. Our goal is not only to select the order of visiting the cities but also to allocate the resources optimally such that the profit per unit of time (the profit rate) is maximized. Although in most TSP variants the weight of an edge connecting two vertices is predefined, this work will assume that the weight of an edge $\{i, j\}$ (representing the travelling time between vertices $i$ and $j$ ) is resource dependent, and the total amount of resource is limited. Our problem is relevant to many engineering and scientific applications where a trade-off between time and cost is required such that the 
primary objective function of the firm (in many cases, maximum profit) is achieved.

The effect of the resource allocation on the travelling (or processing) time is usually given by a resource consumption function. When dealing with sequencing problems, researchers usually assume a linear resource consumption function (e.g. Vickson, 1980; Van Wassenhove and Baker, 1982; Daniels and Sarin, 1989; Janiak and Kovalyov, 1996; Cheng et al, 1998). However, this assumption fails to explain the marginal value product rule in which productivity increases according to the amount of resource at a decreasing rate. Thus, we adopt Monma et al's (1990) resource consumption function to describe the travelling time [Equation (1)], since it is more realistic than the linear one. The travelling time $t_{i j}$ between vertex $i$ and vertex $j$ is expressed as

$$
t_{i j}=\left(\frac{w_{i, j}}{r_{i, j}}\right)^{k}
$$

where $w_{i j}$ is the $i-j$ travelling parameter (a positive value that represents the workload of edge $\{i, j\}) ; r_{i, j}$ is the resource allocated to the edge between vertex $i$ and vertex $j$; and $k$ is a constant positive parameter. Equation (1) has the following properties: each travelling time $\left(t_{i, j}\right)$ is a variable dependent only on the resource allocated $\left(r_{i, j}\right)$ and is a decreasing function of the amount of resource allocated. The marginal travelling time decreases with the increase in the resource consumption, and if no resources are allocated, the travelling time is infinite.

Completing the tour will provide the salesman with a predefined known income $V$. The salesman's profit per unit of time is defined as the profit (calculated by subtracting resource consumption costs from $V$ ) divided by the total time required to complete the tour. The objective of the problem presented in this paper is to maximize the profit per unit of time.

The paper is arranged as follows. Section 2 presents the classic TSP and a formulation of the new problem. Sections 3 and 4 present the solution approach followed by a numerical example. Section 5 presents additional related problems. A summary and conclusions are given in Section 6 .

\section{Problem description and notation}

Let $t_{i, j}$ and $c_{i, j}$ denote the time and the cost (or distance) for travelling from vertex $i$ to vertex $j$, respectively. Let $r_{i, j}$ denote the resource allocated for travelling from vertex $i$ to vertex $j$. Let $R$ denote the total resource consumption per tour. Let $V$ denote the contribution to profit per tour (given in resource units). Let $x_{i, j}$ be a binary decision variable which will receive the value of 1 if the tour passes through the edge connecting vertices $i$ and $j$, and 0 otherwise.

The classic TSP is formulated as follows (P1):

$$
\begin{aligned}
& \text { (P1) } \min Z=\sum_{i=1}^{n} \sum_{j=1}^{n} c_{i, j} x_{i, j} \\
& \text { s.t. } \\
& \sum_{j=1}^{n} x_{i, j}=1 \quad \forall i, i=1,2, \ldots, n \\
& \sum_{i=1}^{n} x_{i, j}=1 \quad \forall j, j=1,2, \ldots, n \\
& x_{i, j}=\{0,1\} \quad \forall i, j \\
& \text { No subtours are allowed }
\end{aligned}
$$

The constraint sets ensure that any selected tour includes all the vertices and that each vertex is visited only once. There are several ways to enforce the last constraint (for eliminating subtours), such as the method proposed by Miller et al (1960).

The objective of the problem descried in this paper is to maximize the profit per unit of time. The profit is calculated by subtracting the resource consumption from $V$; it is then divided by the total time required for completing the tour. Note that the travelling time $t_{i, j}$ varies according to the resource allocation.

This problem is formulated as follows.

$$
\text { (P2) } \min P=\frac{V-\sum_{i=1}^{n} \sum_{j=1}^{n} r_{i, j} x_{i, j}}{\sum_{i=1}^{n} \sum_{j=1}^{n} t_{i, j} x_{i, j}}
$$

s.t.

$\sum_{j=1}^{n} x_{i, j}=1 \quad \forall i, i=1,2, \ldots, n$

$\sum_{i=1}^{n} x_{i, j}=1 \quad \forall j, j=1,2, \ldots, n$

$x_{i, j}=\{0,1\} \quad \forall i, j$

No subtours are allowed

Adopting Monma et al (1990) resource consumption function [Equation (1)], the objective function (the profit rate) can be expressed as

$$
\text { Profit }=\frac{V-R}{T}
$$

where

$$
\begin{gathered}
R=\sum_{i=1}^{n} \sum_{j=1}^{n} r_{i, j} x_{i, j} \\
T=\sum_{i=1}^{n} \sum_{j=1}^{n}\left(\frac{w_{i j}}{r_{i j}}\right)^{k} x_{i, j}
\end{gathered}
$$

In order to maximize the profit rate, we must determine the resource consumption per tour $(R)$, the tour $\left(x_{i, j}\right)$, and the resource allocation $\left(r_{i, j}\right)$. 


\section{Solution approach}

Let us consider any arbitrary feasible tour. The tour can be represented as a chain of connected edges in a series connection (a one-path graph). The optimal resource allocation to minimize the duration of a path in a series-parallel graph was presented by Monma et al (1990).

In our case, any arbitrary feasible tour can be represented as a series of connected graphs were $G_{1}$ represents the edge connecting the first and second vertices along tour, $G_{2}$ represents the edge connecting the second and third vertices along the tour and so on. And therefore $G=G_{1} \rightarrow G_{2} \rightarrow$ $\cdots \rightarrow G_{n}$ represents the entire tour, i.e. the ordered list of edges which constructs the entire tour.

We will first try to solve the following problem in order to determine the optimal resource allocation for a known limited amount of nonrenewable resource $R$

$$
\begin{aligned}
& \operatorname{Min} T=\sum_{j=1}^{n}\left(\frac{w_{j}}{r_{j}}\right)^{k} \\
& \text { s.t. } \\
& \sum_{j=1}^{n} r_{j} \leq R
\end{aligned}
$$

The suitable Lagrange function is:

$$
L\left(r_{1}, \ldots, r_{n}, \lambda\right)=\sum_{j=1}^{n}\left(\frac{w_{j}}{r_{j}}\right)^{k}+\lambda\left(\sum_{j=1}^{n} r_{j}-R\right)
$$

And the sufficient conditions for an optimal solution are:

$$
\begin{aligned}
& \text { I } \frac{\partial L\left(r_{1}, \ldots, r_{n}, \lambda\right)}{\partial r_{j}}=0 \quad \forall j=1, \ldots, n \\
& \text { II } \frac{\partial L\left(r_{1}, \ldots, r_{n}, \lambda\right)}{\partial \lambda}=0
\end{aligned}
$$

Thus, we get:

$$
\frac{\partial L\left(r_{1}, \ldots, r_{n}, \lambda\right)}{\partial r_{j}}=k\left(\frac{w_{j}}{r_{j}}\right)^{k-1} \cdot\left(-\frac{w_{j}}{r_{j}^{2}}\right)+\lambda=0 \rightarrow \lambda=\frac{k\left(\frac{w_{j}}{r_{j}}\right)^{k}}{r_{j}}
$$

Since this condition is identical to all graphs $G_{1}, \ldots, G_{n}$ we can conclude that $\frac{k\left(\frac{w_{i}}{r_{i}^{*}}\right)^{k}}{r_{i}^{*}}=\frac{k\left(\frac{w_{j}}{r_{j}^{*}}\right)^{k}}{r_{j}^{*}}=\cdots=\frac{k\left(\frac{w_{n}}{r_{n}^{*}}\right)^{k}}{r_{n}^{*}}$ Thus, we can say that $r_{i}^{*}=\frac{r_{j}^{*} w_{i}^{\frac{k}{k+1}}}{w_{j}^{\frac{k}{k+1}}}$.

From condition II, we get: $\sum_{i=1}^{n} r_{i}^{*}=R$ and therefore $R=\sum_{i=1}^{n} r_{i}^{*}=\sum_{i=1}^{n} \frac{r_{j}^{*} w_{i}^{\frac{k}{k+1}}}{w_{j}^{\frac{k}{k+1}}}=\frac{r_{j}^{*} \sum_{i=1}^{n} w_{i}^{\frac{k}{k+1}}}{w_{j}^{\frac{k}{k+1}}}$.

The optimal resource allocation for $G_{j}$ in terms of $R$ is:

$$
r_{j}^{*}=\frac{R w_{j}^{\frac{k}{k+1}}}{\sum_{i=1}^{n} w_{i}^{\frac{k}{k+1}}} .
$$

And the optimal time to complete the entire tour is:

$$
\begin{aligned}
T^{*} & =\sum_{j=1}^{n}\left(\frac{w_{j}}{r_{j}^{*}}\right)^{k}=\sum_{j=1}^{n} \frac{w_{j}^{k}}{\left(\frac{R w_{j}^{\frac{k}{k+1}}}{\sum_{i=1}^{n} w_{i}^{k+1}}\right)^{k}}=\frac{\left(\sum_{i=1}^{n} w_{i}^{\frac{k}{k+1}}\right)^{k}}{R^{k}} \sum_{j=1}^{n} \frac{w_{j}^{k}}{w_{j}^{\frac{k^{2}}{k+1}}} \\
& =\frac{\left(\sum_{i=1}^{n} w_{i}^{\frac{k}{k+1}}\right)^{k}}{R^{k}} \sum_{j=1}^{n} w_{j}^{\frac{k}{k+1}}
\end{aligned}
$$

Since $\sum_{i=1}^{n} w_{i}^{\frac{k}{k+1}}=\sum_{j=1}^{n} w_{j}^{\frac{k}{k+1}}$, we can rewrite the equation and get:

$$
\begin{aligned}
T^{*} & =\frac{\left(\sum_{j=1}^{n} w_{j}^{\frac{k}{k+1}}\right)^{k}}{R^{k}} \sum_{j=1}^{n} w_{j}^{\frac{k}{k+1}}=\frac{\left(\sum_{j=1}^{n} w_{j}^{\frac{k}{k+1}}\right)^{k+1}}{R^{k}} \\
& =\left(\frac{\left(\sum_{j=1}^{n} w_{j}^{\frac{k}{k+1}}\right)^{\frac{k+1}{k}}}{R}\right)^{k}
\end{aligned}
$$

For a given total resource consumption $R$, the minimum completion time of $G$ is:

$$
T^{*}=\left(\frac{w_{G}}{R}\right)^{k}
$$

Where the equivalent workload $w_{G}$ is:

$$
w_{G}=\left(\sum_{j=1}^{n} w_{j}^{\frac{k}{k+1}}\right)^{\frac{k+1}{k}}
$$

Rewriting Equation (2) we get:

$$
\text { profit }=\frac{V-R}{T}=\frac{V-R}{\left(\frac{w_{G}}{R}\right)^{k}}=\frac{(V-R) R^{k}}{w_{G}^{k}}
$$

Since $w_{G}$ is independent of $R$, we can derive the profit according to $R$ and get:

$$
\frac{d \text { profit }}{d R}=0 \quad \rightarrow R^{*}=V \frac{k}{(k+1)}
$$

Note that $R^{*}<V$ and therefore the optimal profit is always positive.

Although the profit function is not convex, it is a unimodal function and has a single maximum point (see Figure 1). As can be seen from Equation (9), $R^{*}$ is independent of the selected tour. Moreover, if $R^{*}$ is applied, the profit rate is positive. In the case of an additional constant cost per unit of time, the optimal amount of resource $R^{*}$ will not change.

In order to maximize the profit rate, $w_{G}$ must be minimized. $w_{G}$ is a direct result of the selected tour as can be seen in Equation (7). The tour with the minimal $w_{G}$ is the one that 


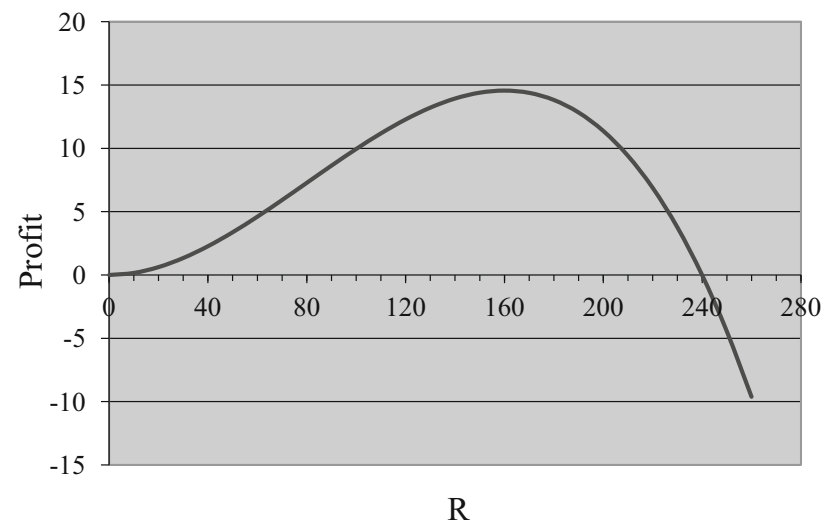

Figure 1 Profit as a function of $R$ where $V=240$ (this graph is typical for $k=2$ ).

minimizes $\sum_{j=1}^{n}\left(w_{j}\right)^{\frac{k}{k+1}}$, and thus, we define new edge weights: $S_{i, j}=\left(w_{i, j}\right)^{\frac{k}{k+1}}$. Now, we can minimize $w_{G}$ by solving $\mathbf{P 1}$, either optimally by any optimal procedure or by any approximation method. The optimization procedure for our problem is formally stated as follows.

Solution Procedure

Step 1 Calculate $R^{*}$ using Equation (9).

Step 2 Set new edge weights $S_{i, j}=\left(w_{i, j}\right)^{\frac{k}{k+1}}$.

Step 3 Solve the TSP (P1) by any known method.

Step 4 For the selected tour:

Calculate $w_{G}$ using Equation (7)

Calculate the optimal resource allocation using Equation (5).

The travelling time and the profit are calculated by Equations (6) and (8), respectively.

We note that if step 3 is solved by a $(1+\varepsilon)$-approximation algorithm, the resulting workload of the tour can be expressed as

$$
w_{g_{a p x}} \leq\left((1+\varepsilon) \sum_{j=1}^{n}\left(w_{j}\right)^{\frac{k}{k+1}}\right)^{\frac{k+1}{k}}=(1+\varepsilon)^{\frac{k+1}{k}} w_{g}^{*}
$$

where $w_{g}^{*}$ denotes the workload of the optimal tour. Therefore, the time of the tour is

$$
T_{a p x}=\left(\frac{w_{g_{a p x}}}{R}\right)^{k} \leq\left(\frac{(1+\varepsilon)^{\frac{k+1}{k}} w_{g}^{*}}{R}\right)^{k}=(1+\varepsilon)^{k+1}\left(\frac{w_{g}^{*}}{R}\right)^{k}
$$

and the approximated value of the profit per unit of time is

$$
P_{\text {approx }}=\frac{V-R}{T_{a p x}} \leq \frac{V-R}{(1+\varepsilon)^{k+1}\left(\frac{w_{g}^{*}}{R}\right)^{k}}=\left(\frac{1}{(1+\varepsilon)^{k+1}}\right) P^{*}
$$

Thus, the whole procedure yields an $\frac{1}{(1+\varepsilon)^{k+1}}$ approximation for P2.

\section{Numerical example}

The following numerical example demonstrates the stages of the procedure for a graph of 5 vertices, where $V=240$ resource units (r.u.) and $k=2$. The objective is to find the tour and the resource allocation that will maximize the profit per unit of time.

Table 1 describes the workload $\left(w_{i j}\right)$ between each pair of vertices.

Solution

Step 1 The optimal amount of the resource is $R^{*}=\frac{2}{2+1} \times$ $240=160$ r.u.

Step 2 New weights for every edge are calculated (Table 2).

Step 3 The optimal tour obtained by solving the TSP (P1) with the new weights optimally is $1 \rightarrow 2 \rightarrow 3 \rightarrow$ $4 \rightarrow 5 \rightarrow 1$.

Step 4 For the optimal tour:

$$
\begin{aligned}
w_{G}= & (4+4+4+4+36)^{\frac{2+1}{2}}=374.98 \\
& r_{1,2}^{*}=\frac{4}{4+4+4+4+36} \times 160=12.3 \text { r.u. } \\
& r_{2,3}^{*}=12.3 \text { r.u. }, r_{3,4}^{*}=12.3 \text { r.u. }, \\
& r_{4,5}^{*}=12.3 \text { r.u., } r_{5,1}^{*}=110.8 \text { r.u. }
\end{aligned}
$$

The travelling time is $T^{*}=(374.98 / 160)^{2}=5.49$.

The optimal profit rate is Profit $=\frac{240-160}{(374.98 / 160)^{2}}=14.56$.

\section{Related problems}

The following two problems are related to $\mathbf{P 2}$ and can be solved by a similar approach.

Problem 3 Minimizing the total travelling time $(T)$ when the amount of resource $(R)$ is limited. The problem is formulated as:

$$
\text { (P3) } \min T=\sum_{i=1}^{n} \sum_{j=1}^{n} x_{i, j}\left(\frac{w_{i, j}}{r_{i, j}}\right)^{k}
$$

subject to the same constraints as the classic TSP (as presented in Section 2), but with an additional resource limit constraint

$$
\sum_{i=1}^{n} \sum_{j=1}^{n} r_{i, j} x_{i, j} \leq R
$$

This problem can arise in many real-life scenarios when the amount of available resources is limited. One example is finding the optimal car speed in rally racing when passing through different types of terrain with a limited amount of fuel. Note that when $R$ is restricted, problems $\mathbf{P 3}$ and $\mathbf{P 2}$ are essentially the same, since $V-$ $R$ is a constant and the objective in $\mathbf{P 2}$ becomes minimizing $T$. 
Table 1 Workload from-to table

\begin{tabular}{llllll}
\hline From & \multicolumn{5}{c}{ To } \\
\cline { 2 - 6 } & 1 & 2 & 3 & 4 & 5 \\
\hline 1 & $\mathbf{x}$ & 8 & 121 & $\mathbf{x}$ & 512 \\
2 & 216 & $\mathbf{x}$ & 8 & 64 & 729 \\
3 & 125 & 1000 & $\mathbf{x}$ & 8 & 1000 \\
4 & 512 & 729 & 512 & $\mathbf{x}$ & 8 \\
5 & 216 & 343 & 27 & 216 & $\mathbf{x}$ \\
\hline
\end{tabular}

Table $2 S_{i j}$ from-to table

\begin{tabular}{llllll}
\hline From & \multicolumn{5}{c}{ To } \\
\cline { 2 - 6 } & 1 & 2 & 3 & 4 & 5 \\
\hline 1 & $\mathbf{x}$ & 4 & 121 & $\mathbf{x}$ & 64 \\
2 & 36 & $\mathbf{x}$ & 4 & 16 & 81 \\
3 & 25 & 100 & $\mathbf{x}$ & 4 & 100 \\
4 & 64 & 81 & 16 & $\mathbf{x}$ & 4 \\
5 & 36 & 49 & 9 & 36 & $\mathbf{x}$ \\
\hline
\end{tabular}

We saw earlier that for $\mathbf{P 2}$ we can calculate the optimal amount of resource $R^{*}$. Opposed to $\mathbf{P 2}$, in $\mathbf{P 3}$ we will always prefer a higher amount of resource, as it will necessarily yield a faster tour. In order to find the minimal $T$, we use the same procedure described in Section 3, but skip the first step.

Problem 4 Minimizing the total resource consumption $(R)$ while not exceeding a predefined travelling time $(T)$. The problem is formulated as:

$$
\text { (P4) } \min R=\sum_{i=1}^{n} \sum_{j=1}^{n} r_{i, j} x_{i, j}
$$

subject to the same constraints as in (P2), but with an additional time limit constraint

$$
\sum_{i=1}^{n} \sum_{j=1}^{n} t_{i, j} x_{i, j} \leq T
$$

This problem may arise in transportation and distribution systems where a task has to be performed within a given amount of time and the objective is to minimize the expenses under this time constraint. P4 is solved by the same procedure described in Section 3 with a slight difference in step 1. The total travelling time of the tour $T_{\text {tour }}=\left(\frac{w_{G}}{R}\right)^{k}$ must satisfy the constraint $T_{\text {tour }} \leq T$. It is easy to see that under the optimal solution, the time constraint is satisfied with equality. Thus, $R \geq w_{G} \sqrt[k]{T}$ and $R^{*}=w_{G} \sqrt[k]{T}$ and therefore the objective becomes to minimize $w_{G}$.

\section{Summary}

This paper introduced a new extension of the classic TSP where the travelling times are resource dependent and the objective is to maximize the profit per unit of time. We used the equivalent load method to find the optimal resource allocation for any tour and found that the optimal amount of resource required, $R^{*}$, is independent of the selected tour and that it is a function of $V$ - the contribution to profit per tour. Then, in order to maximize the profit we had to find the tour with the minimal equivalent workload. This was done by solving the classic TSP by using transformed edge workloads. In addition, the optimal resource allocation for the selected tour and $R^{*}$ were obtained analytically. Notably, the routing complexity remains the same as that of the classic TSP.

\section{References}

Abeledo H, Fukasawa R, Pessoa A and Uchoa E (2013). The time dependent travelling salesman problem: Polyhedra and algorithm. Mathematical Programming Computation 5(1):27-55.

Applegate DL, Bixby R, Chvatal V and Cook WJ (2007). The Traveling Salesman Problem: A Computational Study. Princeton University Press: Princeton, NJ.

Applegate DL, Bixby RE, Chvátal V, Cook W, Espinoza DG, Goycoolea M and Helsgaun K (2009). Certification of an optimal TSP tour through 85,900 cities. Operations Research Letters 37(1):11-15.

Cheng TCE, Janiak A and Kovalyov MY (1998). Bicriterion single machine scheduling with resource dependent processing times. SIAM Journal on Optimization 8(2):617-630.

Cook WJ (2011). In Pursuit of the Traveling Salesman: Mathematics at the Limits of Computation. Princeton University Press: Princeton, NJ.

Cook WJ, Cunningham WH, Pulleyblank WR and Schrijver A (2008). Combinatorial Optimization. Wiley: New York.

Daniels RL and Sarin RK (1989). Single machine scheduling with controllable processing times and number of jobs tardy. Operations Research 37(6):981-984.

Feillet D, Dejax P and Gendreau M (2005). Travelling salesman problems with profits. Transportation Science 39(2):188-205.

Hartley R (1985). Linear and Nonlinear Programming. Ellis Horwood Ltd.: Chichester.

Janiak A and Kovalyov MY (1996). Single machine scheduling subject to deadlines and resource dependent processing times. European Journal of Operational Research 94(2):284-291.

Kaspi M, Zofi M and Teller R (2013). Maximizing the profit per unit of time for the travelling salesman problem. In: ICPR 22nd International Conference on Challenges for Sustainable Operations, Iguassu Falls, Brazil, 2013.

Lawler EL, Lenstra JK, Rinnooy Kan AHG and Shmoys DB (1985). The Travelling Salesman Problem: A Guided Tour of Combinatorial Optimization. Wiley: Chichester.

López-Ibáñez M, Blum C, Ohlmann JW and Thomas BW (2013). The travelling salesman problem with time windows: Adapting algorithms from travel-time to makespan optimization. Applied Soft Computing 13(9):3806-3815.

Monma CL, Schrijver A, Todd MJ and Wei VK (1990). Convex resource allocation problems on directed acyclic graphs: Duality, complexity, special cases and extensions. Mathematics of Operations Research 15(4):736-748. 
Miller CE, Tucker AW and Zemlin RA (1960) Integer programming formulation of the travelling salesman problem. Journal of Association for Computing Machinery 7(4):326-329.

Papadimitriou CH and Steiglitz K (1998). Combinatorial Optimization: Algorithms and Complexity. Dover Publications: Mineola, New York.

Van Wassenhove L and Baker KR (1982) A bicriterion approach to time/cost trade-offs in sequencing. European Journal of Operational Research 11(1):48-54.
Vickson RG (1980). Two single machine sequencing problems involving controllable job processing times. AIIE Transactions 12(3):258-262.

Williams HP (1999). Model Building in Mathematical Programming. Wiley: Chichester.

Received 15 August 2016; accepted 16 November 2016 